\title{
Correlation of Joule Heating and Electromigration-induced Mass Transport within Nanoscale Co Interconnects by In Situ STEM
}

\author{
Brian Zutter ${ }^{1}$, Matthew Mecklenburg ${ }^{2}$, Ho Leung Chan ${ }^{1}$ and B. C. Regan ${ }^{1}$ \\ ${ }^{1}$ Department of Physics \& Astronomy, University of California, Los Angeles, Los Angeles, California, \\ United States, ${ }^{2}$ University of Southern California, Los Angeles, California, United States
}

Cobalt is a potential replacement for the copper in highly-scaled local interconnects [1]. Although cobalt has a higher bulk resistivity than copper, its carrier mean-free path is shorter, making it less resistive (and thus more resistant to electromigration (EM) failure) in nanoscale interconnects [2]. Studying EM failure in nanoscale interconnects is challenging, since few techniques can precisely measure EM-induced changes in thickness on the nanoscale [3]. In addition, EM is strongly temperature-dependent, but measuring temperature with nanoscale spatial resolution is difficult. Changes in temperature from Joule heating can be simulated, but this approach is subject to inaccuracies due to the significant uncertainty in the thermal conductivities of thin-film cobalt and thin-film silicon nitride. We use in situ STEM electron energy loss spectroscopy (EELS) to simultaneously measure temperature and mass transport within cobalt nanowires with nanoscale spatial resolution.

Cobalt nanowires (30-nm-thick) are defined via e-beam lithography on electron-transparent 20-nm-thick silicon nitride membranes (Figure 1a). A 4-wire geometry is used, with the sense leads located close to the center of the wire, to provide accurate measurements of changes in resistivity induced by temperature or EM. The nanowires are biased in situ using an electrical biasing holder within a JEOL JEM-2100F operated at $80 \mathrm{kV}$, and EELS spectra are acquired using a Gatan Quantum SE spectrometer.

Temperature can be measured with nanoscale spatial resolution using plasmon-energy expansion thermometry (PEET) [4]. EELS spectra (Figure 1b) are acquired at each pixel in the $270 \times 53$ spectrum image (Figure 2a). Changes in plasmon energy are converted to changes in temperature, using a known coefficient of thermal expansion (CTE). Previously PEET has been demonstrated in materials such as aluminum and silicon $[4,5]$; here we add cobalt to the list. Temperature profiles are plotted versus distance along the wire (Figure 2b) for the forward and reverse current directions. Importantly, the temperature profiles for forward and reverse bias agree closely, as expected since Joule heating is proportional to the square of current density. These profiles show that at these large current densities of $6.7 \times 10^{11} \mathrm{~A} / \mathrm{m}^{2} \mathrm{Joule}$ heating is significant (over $300^{\circ} \mathrm{C}$ ), and thus may influence EM.

Changes in cobalt thickness can be determined from the same low-loss EELS data used to compute temperatures. The intensity of the zero loss peak is related to the intensity of the plasmon resonances by a Poisson distribution [6], thus the cobalt thickness can be computed from the low-loss spectrum to the extent that the supporting silicon nitride membrane can be ignored. EELS data is acquired before, during, and after 17 mins of biasing. Small changes in thickness are measured between the before-bias and afterbias images (Figure 2c). Importantly, Co is removed from the anode in both bias directions, indicating EM. Loss of material from the anode is consistent with a positive wind-force coefficient, $Z^{*}$. This result disagrees with a previous study, which measured a negative $Z^{*}$ in cobalt up to $600{ }^{\circ} \mathrm{C}$ [7]. We plan future experiments with higher current density, different wire geometries, and remote heaters to further understand this discrepancy [8]. 

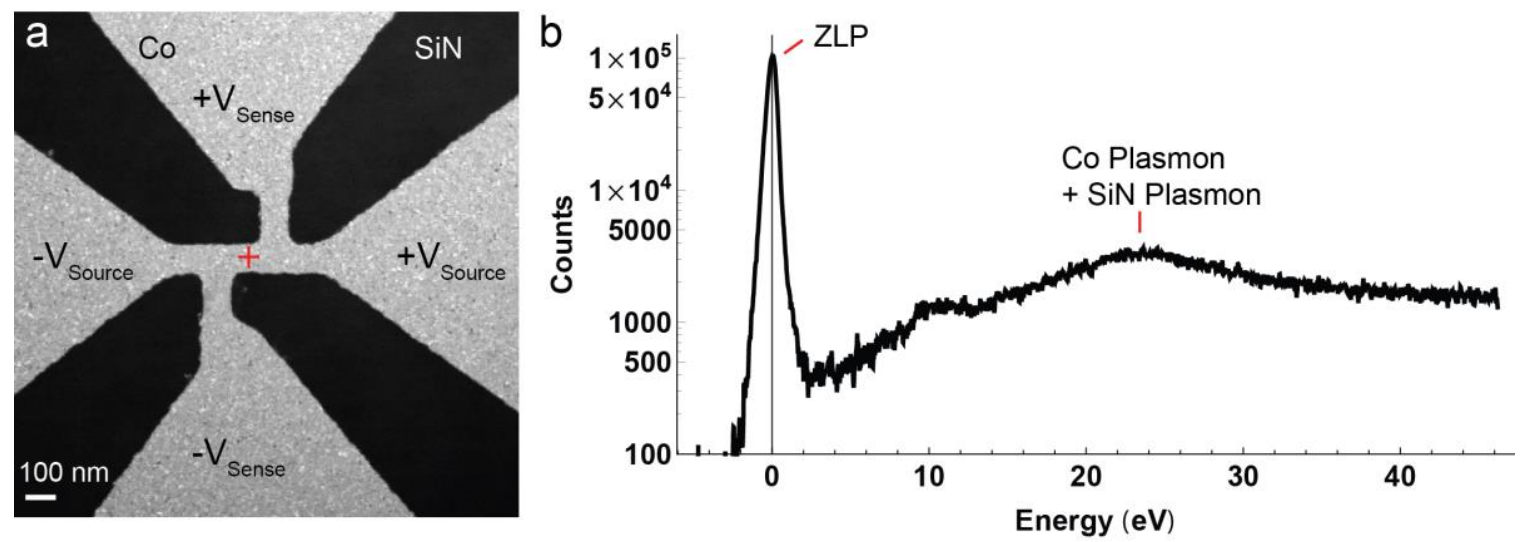

Figure 1. Figure 1. The cobalt device geometry is shown in an ADF STEM image (a). An EELS spectrum (b), taken from the red cross in (a), shows the zero loss peak (ZLP) and the overlapping cobalt and silicon nitride plasmons.

a
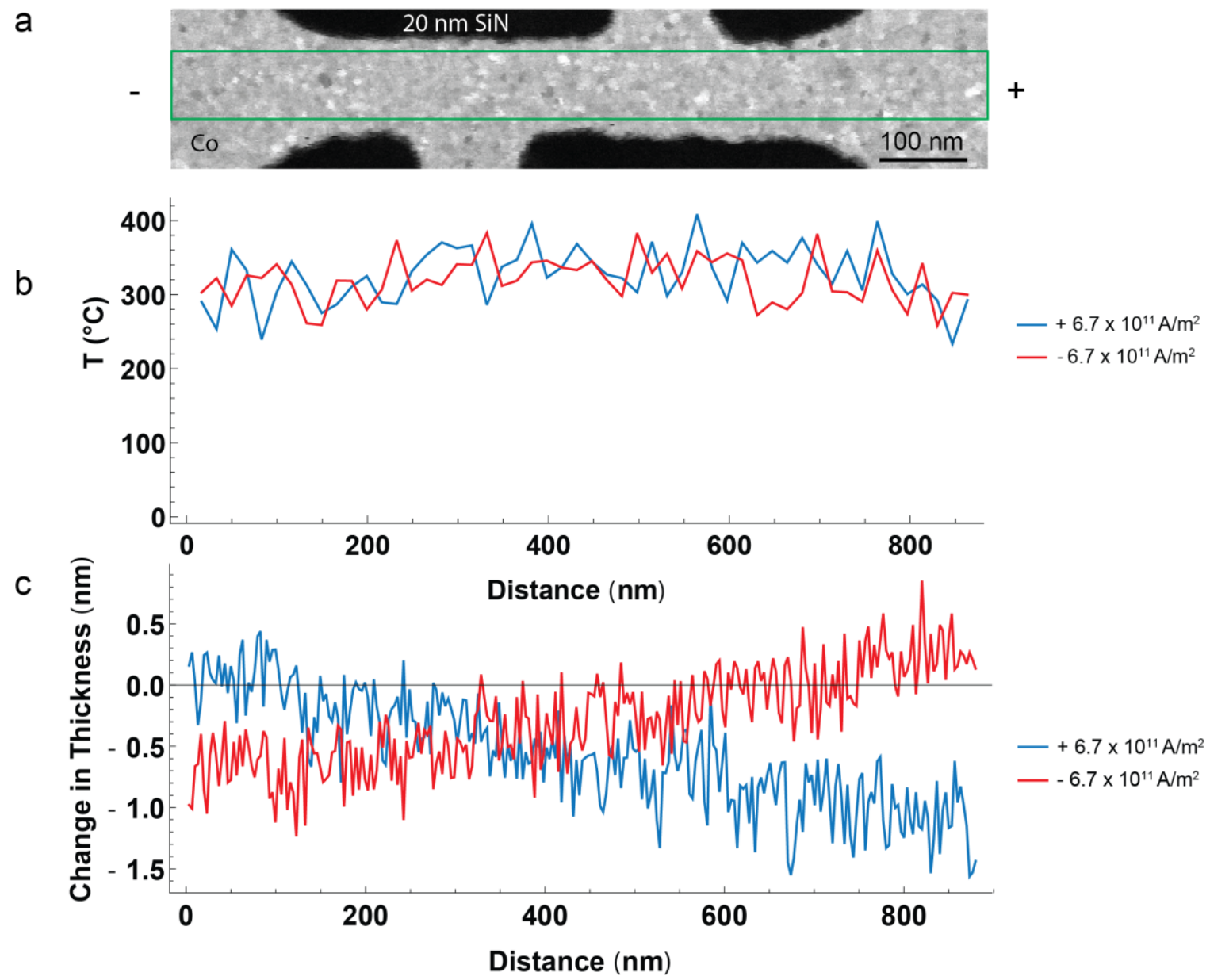

Figure 2. Figure 2. Higher-magnification ADF image (a) of the same Co device in (Figure 1a). The temperature plot (b) is generated by measuring the shift in plasmon energy between the "during bias" data and the "after bias" data. The change in thickness plot (c) is generated by measuring the change in plasmon intensity between the "before bias" and "after bias" data. Plots of temperature and thickness are calculated using EELS data from the region outlined in green in (a). 


\section{References}

[1] F. Griggio et al., IEEE International Reliability Physics Symposium (2018), p. 6E.3 1-5

[2] V. der Veen et al., IEEE International Interconnect Technology Conference (2018), p. 172-174.

[3] B. Engler and R. Hull, Microscopy and Microanalysis. 25 (2019), p. 1902-1903.

[4] Mecklenburg et al., Science. 347 (2015), p. 629-632.

[5] Mecklenburg et al., Physical Review Applied. 9 (2018), p. 014005 1-7.

[6] L. Reimer and H. Kohl, Transmission Electron Microscopy. (2008), p. 171.

[7] G. J. Van Gurp, Thin Solid Films. 38 (1976), p. 295-311.

[8] This work was supported by SRC NMP 2872.001, NSF STC award DMR-1548924 (STROBE), by NSF award DMR-1611036. Data presented here were acquired at the Core Center of Excellence in Nano Imaging (CNI) at the University of Southern California. 